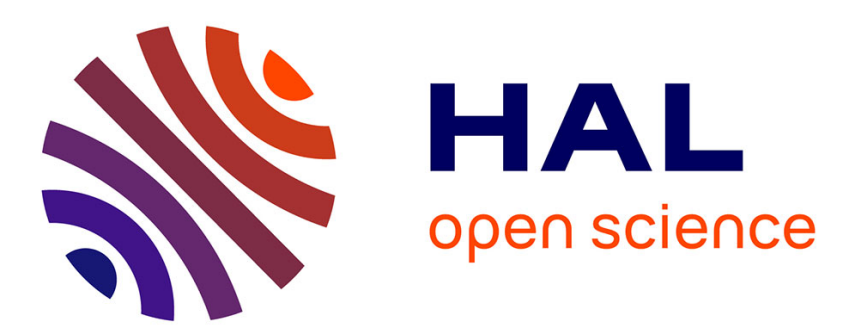

\title{
An analytic approach for the evolution of the static/flowing interface in viscoplastic granular flows
}

François Bouchut, Ioan R. Ionescu, Anne Mangeney

\section{To cite this version:}

François Bouchut, Ioan R. Ionescu, Anne Mangeney. An analytic approach for the evolution of the static/flowing interface in viscoplastic granular flows. Communications in Mathematical Sciences, 2016, 14 (8), pp.2101-2126. 10.4310/CMS.2016.v14.n8.a2 . hal-01081213v3

\section{HAL Id: hal-01081213 \\ https://hal.science/hal-01081213v3}

Submitted on 26 Jan 2016

HAL is a multi-disciplinary open access archive for the deposit and dissemination of scientific research documents, whether they are published or not. The documents may come from teaching and research institutions in France or abroad, or from public or private research centers.
L'archive ouverte pluridisciplinaire HAL, est destinée au dépôt et à la diffusion de documents scientifiques de niveau recherche, publiés ou non, émanant des établissements d'enseignement et de recherche français ou étrangers, des laboratoires publics ou privés. 


\title{
An analytic approach for the evolution of the static/flowing interface in viscoplastic granular flows
}

\author{
François Bouchut*, Ioan R. Ionescuł Anne Mangeney ${ }^{\ddagger \S}$
}

\begin{abstract}
Observed avalanche flows of dense granular material have the property to present two possible behaviours: static (solid) or flowing (fluid). In such situation, an important challenge is to describe mathematically the evolution of the physical interface between the two phases. In this work we derive analytically a set of equations that is able to manage the dynamics of such interface, in the thin-layer regime where the flow is supposed to be thin compared to its downslope extension. It is obtained via an asymptotics starting from an incompressible viscoplastic model with Drucker-Prager yield stress, in which we have to make several assumptions. Additionally to the classical ones that are that the curvature of the topography, the width of the layer, and the viscosity are small, we assume that the internal friction angle is close to the slope angle (meaning that the friction and gravity forces compensate at leading order), the velocity is small (which is possible because of the previous assumption), and the pressure is convex with respect to the normal variable. This last assumption is for the stability of the double layer static/flowing configuration. A new higher-order non-hydrostatic nonlinear coupling term in the pressure allows us to close the asymptotic system. The resulting model takes the form of a formally overdetermined initial-boundary problem in the variable normal to the topography, set in the flowing region only. The extra boundary condition gives the information on how to evolve the static/flowing interface, and comes out from the continuity of the velocity and shear stress across it. The model handles arbitrary velocity profiles, and is therefore more general than depth-averaged models.
\end{abstract}

Keywords: Granular flows, viscoplastic flows, Drucker-Prager yield stress, static/flowing transition, interface dynamics, non-hydrostatic pressure

\section{Introduction}

One of the key features of dense granular materials, like sand or dust, is to flow like a fluid or behave like a solid, depending on the balance between on one hand the driving forces related for instance to gravity, free surface slope or inflow velocity, and on the other hand a threshold related to the frictional properties of the material and to its interaction with the substrate or container. Describing the transition between the so-called flowing and static states is critical for industrial and geophysical purposes. In particular, the static/flowing transition plays a key role in erosion-deposition processes within geophysical flows such as debris flows or debris and snow avalanches [29, 49, 42, 43, 33, 22].

The static/flowing transition is closely related to the rheology of dense granular materials. While this rheology has been very much studied, e.g. $[2,44]$, the accurate description of

\footnotetext{
*Université Paris-Est, Laboratoire d'Analyse et de Mathématiques Appliquées (UMR 8050), CNRS, UPEM, UPEC, F-77454, Marne-la-Vallée, France (francois.bouchut@u-pem.fr)

${ }^{\dagger}$ CNRS \& Université Paris 13, Laboratoire des Sciences des Procédés et des Matériaux, 99, Av. J.-B. Clement, F-93430 Villetaneuse, France (ioan.r.ionescu@gmail.com)

$\ddagger$ Université Paris Diderot, Sorbone Paris Cité, Institut de Physique du Globe de Paris, Equipe de Sismologie, 1 rue Jussieu, 75005 Paris, France (mangeney@ipgp.fr)

§INRIA, CEREMA, Lab. J.-L. Lions, Equipe ANGE, Paris, France
} 
the static/flowing transition is still an open issue. In particular, depending on the scale of description, this transition can be viewed as a sharp or as a more continuous change of states. Indeed, it has been shown that for surface flows over an erodible substrate, the velocity decreases exponentially from the flowing to the static state, with creeping and intermittent motion of the grains near the static state [46]. At the "large scale" (i.e. the scale of the flow), this transition has been described by introducing a Drucker-Prager [35, 37, 18, 32] or a Coulomb [19] yield stress or by describing explicitly the transition from the flowing to the static states through an order parameter that varies continuously between these two states $[4,5,42]$.

As proposed by [35], dense granular flows can be described by an incompressible viscoplastic law with Drucker-Prager yield stress. The so-called $\mu(I)$ rheology, where $I$ is the inertial number, involves a viscosity that depends on the pressure and the strain rate experienced by the granular material. It enables to quantitatively reproduce granular column collapse over horizontal and inclined slopes and granular flow experiments over erodible substrate [35, 32]. Furthermore, this continuum approach is in good agreement with discrete element simulations of granular column collapse [37]. Reference [32] showed that using a constant viscosity gives very similar results to using the $\mu(I)$ rheology for granular column collapses of small aspect ratios over horizontal and inclined planes. In all these regimes, the basic ingredients, required to reproduce dry granular flows at the "large scale" from the destabilization phase to the arrest phase, seem to be present in the proposed viscoplastic rheologies. However, the precise description of the yield stress, of the viscosity as well as the possible role of compressibility are still open and challenging questions, e.g. [28, 32, 38]. Furthermore, recent studies show that these models, and in particular the $\mu(I)$ rheology, are ill-posed for small and large values of the inertial number [8]. This is the case near the static/flowing transition and in particular in some regions within the granular mass during granular column collapse simulated by [37] and [32]. Reference [8] suggests that the good results obtained by [37] despite the ill-posedness of the problem may be due to the use of their regularization method and to the finite pressure they imposed at the free surface. However, [32] also obtained very good results by using an augmented Lagrangian method with no specific condition on the surface pressure. As suggested by [8], these good results may instead be due to the use of a coarse mesh such that simulations avoid the effect of the ill-posedness by the damping of the faster growing high wavenumbers.

Based on these results, we propose to start here from the most simple viscoplastic model, based on incompressibility, constant viscosity and Drucker-Prager yield stress, as tested in [32]. We thus consider the dynamics of an incompressible non-Newtonian material described by the equations:

$$
\begin{gathered}
\operatorname{div} \mathbf{U}=0, \\
\partial_{t} \mathbf{U}+\mathbf{U} \cdot \nabla \mathbf{U}=\operatorname{div} \boldsymbol{\sigma}-\mathbf{g},
\end{gathered}
$$

where $\mathbf{U}$ is the velocity vector, $-\mathbf{g}$ is the gravity force, and $\boldsymbol{\sigma}$ is the stress tensor normalized by the density, that is assumed to be constant. The tensor $\boldsymbol{\sigma}$ is symmetric, and we assume a viscoplastic behaviour described by the relation

$$
\boldsymbol{\sigma}=-p \mathrm{I} d+2 \nu D \mathbf{U}+\kappa \sqrt{2} \frac{D \mathbf{U}}{\|D \mathbf{U}\|},
$$

where $p$ is the scalar pressure, $D \mathbf{U}$ is the strain rate tensor,

$$
D \mathbf{U}=\frac{\nabla \mathbf{U}+(\nabla \mathbf{U})^{t}}{2}
$$

the coefficient $\nu \geq 0$ is the kinematic viscosity, and $\kappa \geq 0$ is the yield stress. Here the norm of a matrix $A=\left(A_{i j}\right)$ is the Frobenius norm $\|A\|=\left(\sum_{i j} A_{i j}^{2}\right)^{1 / 2}$. We do not put a factor $1 / 2$ in the definition of this norm, as many authors do, and it leads to the normalization factor $\sqrt{2}$ in (1.3). The important feature in (1.3) is that the stress $\boldsymbol{\sigma}$ is multivalued when 
$D \mathbf{U}=0$. This means that (1.3) has to be understood in rigorous terms as

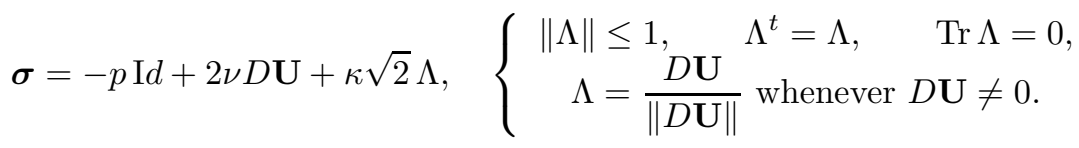

In general, in (1.3), $\nu$ and $\kappa$ can be rate and pressure dependent, i.e. they can depend on $\|D \mathbf{U}\|$ and $p$. Note however that then different couples $(\nu, \kappa)$ can correspond to the same law, since $\boldsymbol{\sigma}$ in (1.3) only depends on the sum $2 \nu\|D \mathbf{U}\|+\kappa \sqrt{2}$. With these notations, the constant yield stress relation $\kappa(\|D \mathbf{U}\|=0, p)=$ cst characterizes the Bingham model. On the contrary, assuming that a free surface separates the material from air at the reference zero pressure, the Drucker-Prager yield stress [20] can be written

$$
\kappa(\|D \mathbf{U}\|=0, p)=\mu_{s} p
$$

where $\mu_{s} \geq 0$ is the static internal Coulomb friction coefficient, a constant depending only on the material $\left(\mu_{s}=\tan \delta\right.$, where $\delta$ is the internal friction angle of the material). This relation (1.6) does not depend on the choice of the couple $(\nu, \kappa)$. In order to get uniqueness of $\nu$ and $\kappa$ in the decomposition (1.3), we adopt the convention of [32] that $\kappa \equiv \kappa(p)$ and $\nu(\|D \mathbf{U}\|, p)\|D \mathbf{U}\| \rightarrow 0$ as $\|D \mathbf{U}\| \rightarrow 0$. This means that the last term in (1.3) is the rate independent (pure plastic) part of the law. Taking into account an eventually negative pressure, the Drucker-Prager yield stress (1.6) is thus written in this particular decomposition

$$
\kappa \equiv \kappa(p)=\mu_{s} \max (p, 0) .
$$

Concerning the viscosity $\nu$, as commented above it is taken constant in this work, although the value

$$
\nu=\left(\mu(I)-\mu_{s}\right) \frac{\max (p, 0)}{\sqrt{2}\|D \mathbf{U}\|}
$$

should be preferred generally, corresponding to the $\mu(I)$ rheology of [35], with $I \sim\|D \mathbf{U}\| / \sqrt{p}$. Taking into account $\mu(I)$ may be very important in some situations, but would lead here to some complications hiding the main features of our approach. Note that [35] proposes the choice $\nu=0, \kappa=\mu(I) \max (p, 0)$, which is equivalent to the choice (1.8), (1.7) (see [32]). The Drucker-Prager yield stress (1.6) provides a better approximation of the behaviour of granular flows than the Bingham yield stress [35]. In particular, while in Bingham fluids, a plug zone may develop on top of the flowing layer [6], for Drucker-Prager or Coulomb fluids, the static zone is generally found near the base of the flow due to the specific form of the $\kappa$ law. However, contrary to the Bingham model (studied mathematically by [21] in the viscous case and by [39] in the two-dimensional viscous or inviscid cases) that is always well-posed even without viscosity [13], the Drucker-Prager yield stress with viscosity is ill-posed in some situations, as discussed above.

The system (1.1)-(1.3), (1.7) describes a flowing behaviour where $D \mathbf{U} \neq 0$, and a static behaviour where $D \mathbf{U}=0$. However, the interface between these two domains is not written explicitly, it is embedded in the whole formulation. The aim of this work is to derive a more explicit description of the evolution of this interface in the context of a thin-layer approximation.

Solving the complete set of equations for an incompressible viscoplastic material requires high computational time, preventing applications to natural geophysical flows. As a result, several attempts have been made to reduce the computational cost, essentially based on socalled thin-layer (or shallow water) depth-averaged models. They have been derived rather precisely for Newtonian flows, and studies for non-Newtonian flows have been proposed in $[47,6,7,9,50,25,17,24,45,11,30,31,27,12]$. In these works, the approach is most of the time to average the equations (1.1), (1.2) with respect to a variable normal to the bottom topography, and to close the equations by an assumption on the dependency on this normal variable, motivated by observations from natural or experimental flows. However, doing this, the information contained in (1.2) on the evolution of the full dependence in the normal 
variable is lost, and is replaced by the external input on the profile. Thus by this method, the obtained depth-averaged system can have no relation to solutions of the original system (1.1), (1.2). There are however cases where the profile can be shown compatible with the original system. Numerical methods for non-Newtonian depth-averaged models are described in $[17,1,23]$.

No static/flowing interface exists in the above cited works on depth-averaged models, meaning that either the whole layer of material flows, either it is fully static. The key issue for describing a static/flowing interface in this context is the knowledge of an equation on the static/flowing interface. Defining an equation for this interface in thin-layer models has been done based on phenomenological models or on strong assumptions such as specified velocity profile or reducing the flow to a sliding block [3, 10, 16, 26, 36, 4, 42, 5, 14, 33]. A review is proposed in [34], together with a discussion on the dependency of the interface equation in terms of averaged quantities.

In this work we propose a new approach for describing the static/flowing interface dynamics. It is analytic, in the sense that no phenomenology is put in the interface equation. Rather, the information on the profile and on the static/flowing interface is deduced from the viscoplastic modelling, by asymptotic expansion. Several assumptions are necessary, in particular a thin-layer assumption. Moreover, the viscosity has to be small (meaning that the plastic effects are dominant), the slope of the topography has to be nonzero, the internal friction coefficient has to be close to the slope, the velocity has to be moderate, and the pressure has to be convex with respect to the normal variable, which is a kind of interface stability condition. As in [11], we have to push the expansions to higher order than usually in thin-layer models in order to close the system. In particular, the pressure includes several non-hydrostatic terms. The obtained model is not a depth-averaged model, since the normal variable is still present, and cannot be avoided. It is written as a formally overdetermined boundary problem in the flowing region, for which the extra boundary condition (with respect to a standard parabolic problem) comes from the continuity of the velocity and shear stress across the interface, and drives the evolution of the interface between the two phases. This closed mathematical formulation replaces a formulation by an ordinary differential equation on the interface, frequently proposed in previous works, based on external input or heuristics.

The outline of the paper is as follows. In Section 2 we write down our starting viscoplastic model in topography-based coordinates, and exhibit some reference simple shear solutions. In Section 3 we perform a preliminary thin-layer expansion, that is completed in Section 4 with our main result Theorem 4.1 that states our slow flow model. Particular steady state solutions are given, and a discussion on the interface dynamics is provided. A conclusion is given in Section 5. An appendix is devoted to a key technical argument.

\section{Two-dimensional model and topography-based coor- dinates}

\subsection{Flow domain and boundary conditions}

We consider a viscoplastic material described by the system (1.1)-(1.3), (1.7), set in the spatial domain $x \in \Omega_{t}$ between a fixed bottom topography and a free surface. The system is completed with the following boundary conditions. At the bottom we set the no slip condition

$$
\mathbf{U}=0 \text { at the bottom, }
$$

at the free surface we set the stress free condition

$$
\boldsymbol{\sigma} \mathbf{N}=0 \text { at the free surface, }
$$

where $\mathbf{N}$ is normal to the free surface. Moreover, the free surface evolves with the material, and thus satisfies the kinematic condition

$$
N_{t}+\mathbf{N} \cdot \mathbf{U}=0 \text { at the free surface, }
$$


where $\left(N_{t}, \mathbf{N}\right)$ is a time-space normal to the free surface.

It is not clear if (2.1) leads to a formally well-posed problem if $\nu=0$. Nevertheless, we shall look for solutions for which $\mathbf{U}$ vanishes identically in a neighborhood of the topography (static part), as stated in the last paragraph of Subsection 2.2, thus this condition will be automatically satisfied.

\subsection{Two-dimensional formulation}

We consider the two-dimensional problem. Following $[15,14]$, the topography can be described by the relation $z=B(x)$ in horizontal/vertical coordinates $(x, z)$, where $B(x)$ is a smooth function. We denote by $\theta,-\pi / 2<\theta<\pi / 2$, the angle between the horizontal and the tangent to the topography, and by $X$ the curvilinear coordinate along the topography, so that

$$
\tan \theta=\frac{d B}{d x}, \quad \frac{d X}{d x}=\frac{1}{\cos \theta}, \quad \frac{d B}{d X}=\sin \theta .
$$

We shall consider that $B, \theta, x$ are functions of $X$, instead of $B, \theta, X$ being functions of $x$. The case of affine topography $B$ in terms of $x$, i.e. slope angle $\theta=c s t$, is possible in particular.

A point $M$ within the material layer $\Omega_{t}$ can be described by its distance $Z$ to the topography and the coordinate $X$ of its projection on the topography, or equivalently by the relation

$$
M \equiv\left(x_{M}, z_{M}\right)=(x-Z \sin \theta, B+Z \cos \theta),
$$

where $x, X, B, \theta$ are related by the relations (2.4), as illustrated on Figure 1. The value of

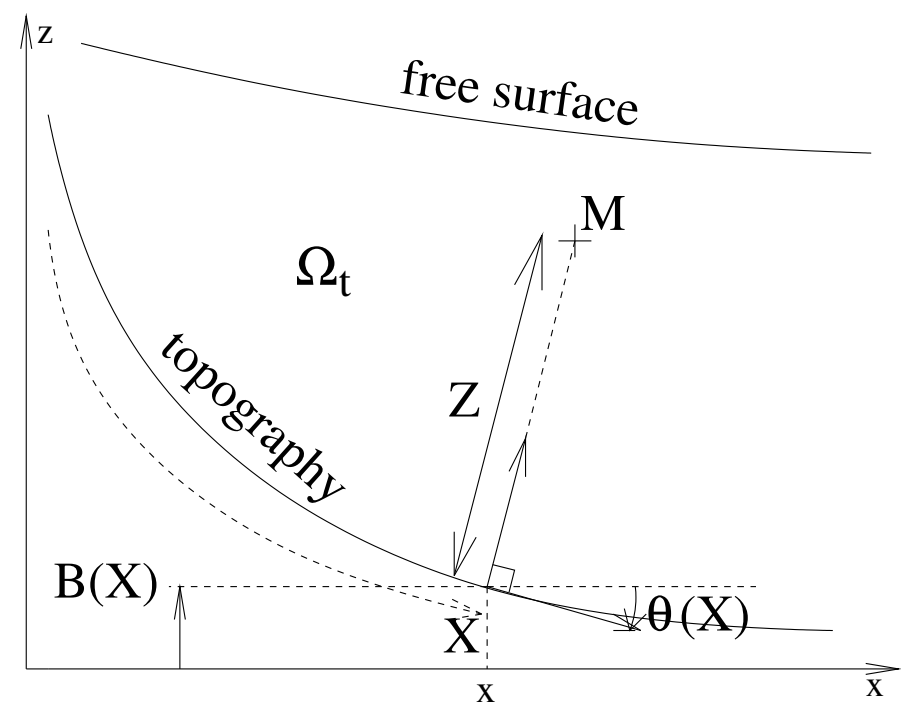

Figure 1: The material layer between topography and free surface.

$Z$ is less than the height $h(t, X)$ of the material in the normal direction,

$$
0<Z<h(t, X) .
$$

The change of variable between $M$ and $(X, Z)$ has Jacobian matrix

$$
\nabla_{X, Z} M=\left(\begin{array}{rr}
J \cos \theta & -\sin \theta \\
J \sin \theta & \cos \theta
\end{array}\right)=\left(\begin{array}{rr}
\cos \theta & -\sin \theta \\
\sin \theta & \cos \theta
\end{array}\right)\left(\begin{array}{ll}
J & 0 \\
0 & 1
\end{array}\right),
$$

with Jacobian determinant

$$
J=1-Z d_{X} \theta=\operatorname{det}\left(\nabla_{X, Z} M\right)
$$


Thus it is a diffeomorphism provided that $h d_{X} \theta<1$, where $d_{X}$ denotes the derivative with respect to the single variable $X$. Note that $d_{X} \theta \equiv d \theta / d X$ is the curvature of the topography. We define the velocity components $(U, W)$ at $M$ in the coordinates tangent and normal to the topography

$$
\left(\begin{array}{c}
U \\
W
\end{array}\right)=\left(\begin{array}{cc}
\cos \theta & \sin \theta \\
-\sin \theta & \cos \theta
\end{array}\right) \mathbf{U}
$$

and the new stress tensor

$$
\boldsymbol{\Sigma}=\left(\begin{array}{cc}
\Sigma_{X X} & \Sigma_{X Z} \\
\Sigma_{Z X} & \Sigma_{Z Z}
\end{array}\right)=\left(\begin{array}{cc}
\cos \theta & \sin \theta \\
-\sin \theta & \cos \theta
\end{array}\right) \boldsymbol{\sigma}\left(\begin{array}{cc}
\cos \theta & -\sin \theta \\
\sin \theta & \cos \theta
\end{array}\right)
$$

with $\Sigma_{X Z}=\Sigma_{Z X}$.

Lemma 2.1 (curvilinear coordinates) The viscoplastic system with Drucker-Prager yield stress (1.1)-(1.3), (1.7) (with $\mu_{s}, \nu$ nonnegative constants) completed by the boundary conditions (2.1)-(2.3) can be written as follows in the coordinates $(t, X, Z)$ defined above, in the unknowns $U(t, X, Z), W(t, X, Z)$ tangential/normal velocity components, $h(t, X)$ normal width of the domain, $\boldsymbol{\Sigma}(t, X, Z)$ stress tensor.

The incompressibility and momentum equations (1.1), (1.2) become

$$
\begin{aligned}
& \partial_{X} U+\partial_{Z}(J W)=0, \\
& \partial_{t}(J U)+U \partial_{X} U+J W \partial_{Z} U+\partial_{X}\left(g(B+Z \cos \theta)-\Sigma_{X X}\right)-\partial_{Z}\left(J \Sigma_{X Z}\right) \\
& =\left(-\Sigma_{X Z}+U W\right) d_{X} \theta, \\
& \partial_{t}(J W)+U \partial_{X} W+J W \partial_{Z} W-\partial_{X} \Sigma_{X Z}+J \partial_{Z}\left(g Z \cos \theta-\Sigma_{Z Z}\right) \\
& =\left(\Sigma_{X X}-\Sigma_{Z Z}-U^{2}\right) d_{X} \theta,
\end{aligned}
$$

where $g>0$ is the gravity constant and $J$ is defined by (2.8). The kinematic free surface condition (2.3) becomes mass conservation

$$
\partial_{t}\left(h-\frac{h^{2}}{2} d_{X} \theta\right)+\partial_{X}\left(\int_{0}^{h} U d Z\right)=0 .
$$

The boundary conditions (2.1), (2.2) become

$$
\begin{aligned}
J \Sigma_{X Z}-\Sigma_{X X} \partial_{X} h=0, \quad J \Sigma_{Z Z}-\Sigma_{X} \partial_{X} h & =0, & & \text { at the free surface } Z=h, \\
W=0, \quad U & =0, & & \text { at the bottom } Z=0 .
\end{aligned}
$$

Finally, the rheological relations (1.3), (1.7) become

$$
\begin{gathered}
\Sigma_{X X}=-p-\left(2 \nu+\frac{\kappa}{\|D \mathbf{U}\| / \sqrt{2}}\right) \partial_{Z} W, \quad \Sigma_{Z Z}=-p+\left(2 \nu+\frac{\kappa}{\|D \mathbf{U}\| / \sqrt{2}}\right) \partial_{Z} W \\
\Sigma_{X Z}=\left(2 \nu+\frac{\kappa}{\|D \mathbf{U}\| / \sqrt{2}}\right) \frac{1}{2}\left(\partial_{Z} U+\frac{\partial_{X} W+U d_{X} \theta}{J}\right) \\
\frac{1}{2}\|D \mathbf{U}\|^{2}=\left(\partial_{Z} W\right)^{2}+\frac{1}{4}\left(\partial_{Z} U+\frac{\partial_{X} W+U d_{X} \theta}{J}\right)^{2}
\end{gathered}
$$

where $\partial_{X} U$ and $\partial_{Z} W$ are related by (2.11), and with

$$
\kappa=\mu_{s} \max (p, 0) .
$$

It has to be understood in (2.17), (2.18) that the vector $\left(\partial_{Z} W, \frac{1}{2}\left(\partial_{Z} U+\frac{\partial_{X} W+U d_{X} \theta}{J}\right)\right) \sqrt{2} /\|D \mathbf{U}\|$ is multivalued in a sense similar to (1.5), that is whenever $\|D \mathbf{U}\|=0$ this vector takes an arbitrary value of norm at most 1 . 
Proof. The change of variables computations were performed in [14] in the more general case of a time-dependent topography $B$. According to this reference, the incompressibility and momentum equations (1.1), (1.2) are transformed into (2.50)-(2.52) in [14]. In our case with time-independent topography one has $\widehat{\partial}_{t} X=\widehat{\partial}_{t} Z=0$ (notations of [14]), thus these equations simplify to (2.11)-(2.13). We do not reproduce here these computations because they are quite messy. They hold whatever is the stress tensor $\boldsymbol{\sigma}$, that transforms into $\boldsymbol{\Sigma}$ according to (2.10). These equations (2.11)-(2.13) were also written down in [15] in the particular case of diagonal stress. Then, again according to [14], the kinematic condition (2.3) transforms into (2.71) in that reference, that is

$$
J_{h} \partial_{t} h+U_{h} \partial_{X} h=J_{h} W_{h}
$$

where the index $h$ means that the quantity is evaluated at $Z=h(t, X)$. As usual in depthaveraging procedures, we integrate (2.11) for $Z$ between 0 and $h(t, X)$ and use

$$
\int_{0}^{h} \partial_{X} U d Z=\partial_{X} \int_{0}^{h} U d Z-U_{h} \partial_{X} h
$$

and the boundary condition $W=0$ at the bottom from (2.16). Noticing with the value (2.8) of $J$ that $\partial_{t}\left(h-\frac{h^{2}}{2} d_{X} \theta\right)=J_{h} \partial_{t} h$, the condition (2.21) can be written equivalently as (2.14), as in [15].

Next, the boundary condition (2.1) becomes obviously (2.16). Concerning the free surface, let us denote by $\left(n_{t}, n\right)$ a time-space normal to the free surface in the variables $t, X, Z$, and recall the notation from $(2.3)$ that $\left(N_{t}, \mathbf{N}\right)$ is a time-space normal in the variables $t, M$ (i.e. in the variables $t, x_{M}, z_{M}$ with $\left.M=\left(x_{M}, z_{M}\right)\right)$. Then according to the change of variables, one can take

$$
\left(n_{t}, n\right)=\left(N_{t},\left(\nabla_{X, Z} M\right)^{t} \mathbf{N}\right)
$$

while since the free surface is defined by the equation $Z-h(t, X)=0$ one can take

$$
\left(n_{t}, n\right)=\left(-\partial_{t} h,-\partial_{X} h, 1\right) .
$$

Note that with (2.7) and (2.9) we get $\left(\nabla_{X, Z} M\right)^{-1} \mathbf{U}=(U / J, W)$, thus we get again that the kinematic condition (2.3) transforms into (2.21). According to (2.23) and (2.7) we have

$$
\mathbf{N}=\left(\nabla_{X, Z} M\right)^{-t} n=\left(\begin{array}{rr}
\cos \theta & -\sin \theta \\
\sin \theta & \cos \theta
\end{array}\right)\left(\begin{array}{ll}
J^{-1} & 0 \\
0 & 1
\end{array}\right) n
$$

thus the stress free condition $\boldsymbol{\sigma} \mathbf{N}=0$ at the free surface from (2.2) transforms with (2.10) into

$$
\Sigma\left(\begin{array}{ll}
J^{-1} & 0 \\
0 & 1
\end{array}\right) n=0 \text { at the free surface. }
$$

But $(2.24)$ gives $n=\left(-\partial_{X} h, 1\right)$, thus $(2.26)$ is exactly $(2.15)$.

Finally, we have to check the rheological relations. Inverting the relation (2.9) and differentiating with respect to $M$, we get using that according to (2.7) one has $\partial_{M} X=$ $(\cos \theta, \sin \theta) / J$

$$
\begin{aligned}
\nabla_{M} \mathbf{U}= & \left(\begin{array}{c}
-U \sin \theta-W \cos \theta \\
U \cos \theta-W \sin \theta
\end{array}\right) \otimes\left(\begin{array}{c}
\cos \theta \\
\sin \theta
\end{array}\right) \frac{d_{X} \theta}{J} \\
& +\left(\begin{array}{cc}
\cos \theta & -\sin \theta \\
\sin \theta & \cos \theta
\end{array}\right) \nabla_{X, Z}\left(\begin{array}{c}
U \\
W
\end{array}\right)\left(\nabla_{X, Z} M\right)^{-1}
\end{aligned}
$$

Then multiplying by the matrices in (2.10), we obtain using again (2.7)

$$
\begin{aligned}
& \left(\begin{array}{cc}
\cos \theta & \sin \theta \\
-\sin \theta & \cos \theta
\end{array}\right) \nabla_{M} \mathbf{U}\left(\begin{array}{cc}
\cos \theta & -\sin \theta \\
\sin \theta & \cos \theta
\end{array}\right) \\
= & \left(\begin{array}{c}
-W \\
U
\end{array}\right) \otimes\left(\begin{array}{l}
1 \\
0
\end{array}\right) \frac{d_{X} \theta}{J}+\nabla_{X, Z}\left(\begin{array}{c}
U \\
W
\end{array}\right)\left(\begin{array}{cc}
J^{-1} & 0 \\
0 & 1
\end{array}\right) .
\end{aligned}
$$


We finally take the symmetric part according to the definition (1.4) of $D \mathbf{U}$ to get

$$
\begin{aligned}
& \left(\begin{array}{cc}
\cos \theta & \sin \theta \\
-\sin \theta & \cos \theta
\end{array}\right) D \mathbf{U}\left(\begin{array}{cc}
\cos \theta & -\sin \theta \\
\sin \theta & \cos \theta
\end{array}\right) \\
= & \left(\begin{array}{cc}
\frac{\partial_{X} U-W d_{X} \theta}{J} & \frac{1}{2}\left(\partial_{Z} U+\frac{\partial_{X} W+U d_{X} \theta}{J}\right) \\
\frac{1}{2}\left(\partial_{Z} U+\frac{\partial_{X} W+U d_{X} \theta}{J}\right) & \partial_{Z} W
\end{array}\right) .
\end{aligned}
$$

We have to take into account (2.11), that ensures that this last matrix is symmetric and trace free. Taking the Frobenius norm in (2.29) yields the formula (2.19). Finally with (2.29), the viscoplastic law (1.3) transforms into the new stress (2.10) as (2.17), (2.18). The multivalued interpretation follows from the fact that the space of symmetric trace free matrices endowed with the Frobenius norm identifies with the space of two-dimensional vectors with the usual Euclidian norm. Finally, (1.7) is identical to (2.20).

In the rest of the paper we consider flows having a transition between a static phase at rest $(\mathbf{U}=0)$ above the bottom, and a flowing phase $(D \mathbf{U} \neq 0)$ above, with nonzero shear. Such flows are characterized by the equations (2.11)-(2.20) completed by

$$
\begin{gathered}
U(t, X, Z)=0 \text { for } 0<Z<b(t, X) \\
\left(\partial_{Z} U+\frac{\partial_{X} W+U d_{X} \theta}{J}\right)(t, X, Z) \neq 0 \quad \text { for } b(t, X)<Z<h(t, X),
\end{gathered}
$$

for some interface $b(t, X)$ such that $0<b(t, X)<h(t, X)$. Note that the first line in $(2.30)$ implies that $W$ also vanishes for $Z<b$, because of (2.11) and the bottom boundary condition on $W$ in (2.16). An illustration is proposed on Figure 2.

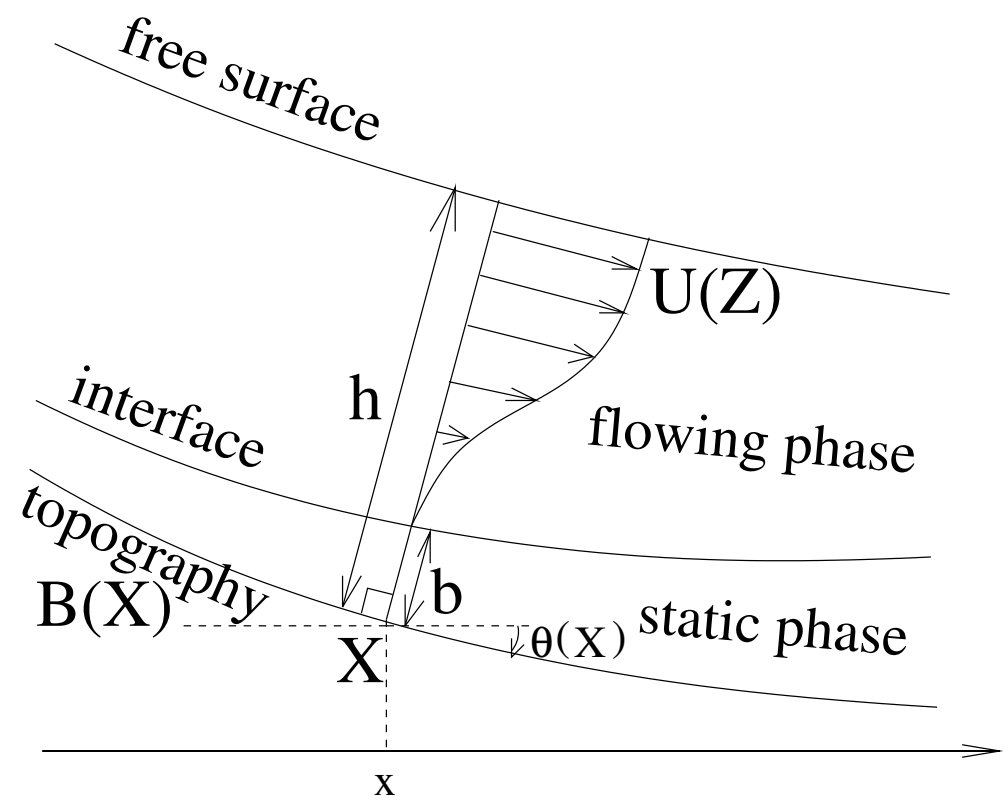

Figure 2: Generic velocity profile for viscoplastic flows with static/flowing interface. The tangential velocity $U$ has a static part $0<Z<b$ and a flowing part $b<Z<h$, separated by an interface $b$, where $h$ is the thickness of the granular layer. The case $\theta<0$ and $\partial_{Z} U>0$ is represented. 


\subsection{Simple shear flows}

In this subsection we show that there exist some particular solutions to the viscoplastic model stated in Lemma 2.1, with a fluid layer flowing above a static layer as stated in (2.30). These solutions exist in the simple shear case, i.e. when the quantities do not depend on $X$, and at least in the inviscid case $\nu=0$.

Proposition 2.2 (Simple shear flows) Assume a constant slope angle $\theta \in(-\pi / 2, \pi / 2)$. Then particular solutions $h, U, W, p$ to (2.11)-(2.20), (2.30) that satisfy

$$
h, U, W, p, \Sigma_{X X}, \Sigma_{Z Z}, \Sigma_{X Z} \text { do not depend on } X,
$$

such that the deviatoric stress is only pure shear (which means that $\boldsymbol{\Sigma}+$ pId has zero diagonal) and that $\theta \partial_{Z} U \leq 0$, are characterized by

$$
W \equiv 0, \quad h=c s t, \quad p=g \cos \theta(h-Z),
$$

and with a sign $s_{b}= \pm 1$ satisfying

$$
s_{b} \theta \leq 0
$$

$U(t, Z)$ has to solve

$$
\partial_{t} U+g \sin \theta+s_{b} \mu_{s} g \cos \theta-\partial_{Z}\left(\nu \partial_{Z} U\right)=0 \text { for } Z>b(t),
$$

where

$$
s_{b} \partial_{Z} U>0 \text { for } Z>b(t)
$$

the boundary conditions

$$
\begin{gathered}
\nu \partial_{Z} U=0 \text { at } Z=h, \\
U=0 \text { at } Z=b(t), \\
\nu \partial_{Z} U=0 \text { at } Z=b(t),
\end{gathered}
$$

and the friction dominating condition

$$
|\tan \theta| \leq \mu_{s}
$$

Proof. Since $\theta$ is constant, the Jacobian $J$ in (2.8) is just $J=1$. Thus (2.11) and the bottom boundary condition (2.16) give that $W \equiv 0$. The mass conservation (2.14) then gives $\partial_{t} h=0$, thus $h$ is constant. The normal momentum conservation equation (2.13) then gives $\partial_{Z}\left(g Z \cos \theta-\Sigma_{Z Z}\right)=0$, and together with the free surface boundary condition on $\Sigma_{Z Z}$ in $(2.15)$ it yields $\Sigma_{Z Z}=-g \cos \theta(h-Z)$. Then, the assumption of pure shear gives that $-\Sigma_{X X}=-\Sigma_{Z Z}=p$ (it is indeed true in the flowing part according to (2.17) since $W=0$, but this has to be assumed in the static part). We deduce that

$$
-\Sigma_{X X}=-\Sigma_{Z Z}=p=g \cos \theta(h-Z) .
$$

The relations (2.18), (2.19) then give

$$
\Sigma_{X Z}=\nu \partial_{Z} U+\kappa \operatorname{sgn}\left(\partial_{Z} U\right)
$$

where the sign is multivalued, i.e. whenever $\partial_{Z} U=0, \operatorname{sgn}\left(\partial_{Z} U\right)$ is any number in $[-1,1]$. It finally remains to write (2.12) by taking into account $(2.4)$,

$$
\partial_{t} U+g \sin \theta-\partial_{Z} \Sigma_{X Z}=0,
$$

and the boundary condition on $\Sigma_{X Z}$ in (2.15),

$$
\Sigma_{X Z}=0 \quad \text { at } Z=h .
$$


This system is completed by the relation (2.20), which is with (2.40)

$$
\kappa=\mu_{s} g \cos \theta(h-Z) .
$$

The considered solutions are thus obtained by finding a solution $U(t, Z)$ defined for $0<Z<h$ satisfying (2.41)-(2.44) and having a static/flowing transition, i.e. $U(t, Z)=0$ for $0<Z<$ $b(t), \partial_{Z} U(t, Z) \neq 0$ for $b(t)<Z<h$, for some $b(t) \in(0, h)$. Applying Lemma A.1 in Appendix (taking $\Phi=g \sin \theta$ for the source, are noting that $\kappa$ is linear, thus convex), we deduce that such solutions $U$ are characterized by a system of equations set in the flowing phase $Z>b$ only, together with boundary conditions and a static equilibrium condition. More precisely, given $s_{b}= \pm 1$ the sign of the shear $\partial_{Z} U$ in the flowing phase, and noting that by assumption (2.33) holds, the problem of finding a solution $U(t, Z)$ defined for $0<Z<h$ to (2.41)-(2.44) and having a static/flowing transition is equivalent to finding a solution $U(t, Z)$ only defined for $b(t)<Z<h$ to (2.34) with (2.35), the boundary conditions (2.36), (2.37), (2.38) and the friction dominating condition (2.39), that must hold for the static layer to exist. This concludes the proof of the statement.

Note that (2.33) means that the slope and the shear rate $\partial_{Z} U$ in the flowing zone have opposite signs, which is very natural, see Figure 2. Here it is important to mention that the boundary condition (2.38) is not imposed a priori, but is a consequence of the analysis provided in Lemma A.1 in Appendix, that states that the shear stress $\Sigma_{X Z}$, and indeed $\nu \partial_{Z} U$, must be continuous through the interface.

The condition (2.39) is really needed. Indeed, if it does not hold, the static/flowing interface can no longer persist for positive time, and we expect in this case the whole layer of material to flow down immediately. Under the condition (2.39), we expect that there is a solution to (2.34)-(2.38). In this system there is one more boundary condition than what is expected in standard parabolic problems (i.e. set in a fixed domain), this is a formally overdetermined problem. This extra condition determines the evolution of the interface $b(t)$.

In the inviscid case $\nu=0$, we can build a solution explicitly.

Proposition 2.3 (Inviscid simple shear solution) Assume that $\theta$ is constant, $\nu=0$, and take $s_{b}=1, \theta \leq 0$ to simplify, satisfying the friction dominating condition (2.39). Then for any initial velocity $U^{0}(Z)$ defined for $0<Z<h$ for some $h>0$ and satisfying $\partial_{Z} U^{0} \geq 0$, $U^{0} \geq 0$, a solution to (2.34)-(2.38) having $U^{0}$ as initial data is given by

$$
U(t, Z)=\max \left(U^{0}(Z)-g\left(\sin \theta+\mu_{s} \cos \theta\right) t, 0\right) \text { for } 0<Z<h,
$$

with interface $b(t)$ defined implicitly by the equation

$$
U^{0}(b(t))=g\left(\sin \theta+\mu_{s} \cos \theta\right) t,
$$

as long as the right-hand side remains less than $U^{0}(h)$. After that time, $b$ can be extended by setting $b(t)=h$, and $U=0$. In particular, the profile of $U$ is the same as the one of $U^{0}$, it is just shifted downwards at constant speed, with negative values clipped.

Proof. Note that because of (2.39) one has $\sin \theta+\mu_{s} \cos \theta \geq 0$. Since $\nu=0$, there remains only the condition $U(t, b(t))=0$ among (2.36)-(2.38) to determine the interface. Then, without the diffusion term, (2.34) is just a differential equation with constant right-hand side in the flowing zone. Its solution is the first argument in the right-hand side of (2.45). It is nondecreasing with respect to $Z$, and indeed the graph of $U^{0}$ is just shifted in the direction of negative velocities, at constant rate. Since $U$ has to remain nonnegative, one has to clip the negative values, which leads to (2.45). According to the interface condition $U(t, b(t))=0$, the interface position $b(t)$ is finally determined by the intersection of the graph of $U$ with the horizontal axis, which yields (2.46). 
We remark that the solution obtained in Proposition 2.3 becomes static in finite time (except if there is equality in (2.39) $|\tan \theta|=\mu_{s}$, in this case the solution is steady). Moreover the interface $b(t)$ is nondecreasing with respect to time, a property that is not expected when there is viscosity. Note that the formula (2.45) works for any (nondecreasing) initial profile $U^{0}$. Thus there is no preferred shape for the $Z$ dependency of the velocity.

\section{Thin-layer asymptotics}

In this section we perform a preliminary thin-layer expansion of the two-dimensional viscoplastic model with Drucker-Prager yield stress. We consider solutions to the model written in curvilinear coordinates (2.11)-(2.20), that include a static/flowing transition (2.30). As is by now common in thin-layer asymptotics [11], we consider a small dimensionless parameter $\varepsilon$ and we assume that

$$
\begin{gathered}
h \sim \varepsilon, \quad d_{X} \theta=O(\varepsilon), \\
\boldsymbol{\Sigma}=O(\varepsilon), \quad p=O(\varepsilon) .
\end{gathered}
$$

These assumptions are related to the knowledge of a characteristic length $L$ and a characteristic time $\tau$ with $L / \tau^{2}=g$, and to the introduction of the appropriate scales. The relation (3.1) means explicitly that $h / L$ is of the order of $\varepsilon$, and that $L d_{X} \theta$ is at most of the order of $\varepsilon$, while (3.2) means that $\Sigma_{X X}, \Sigma_{Z Z}, \Sigma_{X Z}, p$ are at most of the order of $(L / \tau)^{2} \varepsilon$.

We assume that the derivatives with respect to $t$ or $X$ does not induce singularities (they just involve the characteristic scales $L$ and $\tau$, but not $\varepsilon$ ). However, derivatives with respect to $Z$ naturally involve, apart from the scale $L$, a factor $1 / \varepsilon$ at worse, because $Z$ lies in the interval $(0, h)$, which is of order $\varepsilon$. Taking into account the incompressibility $(2.11)$ and the vanishing of $W$ at the bottom in (2.16), for bounded velocities we get the following natural assumptions of orders of magnitude

$$
\begin{gathered}
U=O(1), \quad W=O(\varepsilon) \\
\partial_{X} U=O(1), \quad \partial_{X} W=O(\varepsilon), \\
\partial_{Z} U=O(1 / \varepsilon), \quad \partial_{Z} W=O(1) .
\end{gathered}
$$

Now and further on, we do not write explicitly the units in terms of $L$ and $\tau$, but only the orders of magnitude in terms of $\varepsilon$. Note that another scaling which is used in thin-layer granular flows consists in taking the free-fall time scale $\sqrt{h / g}$, which is in $\sqrt{\varepsilon}$, leading to $U \simeq \sqrt{g h}$, which is also of order $\sqrt{\varepsilon}$.

It would be desirable to make an expansion of the viscoplastic model with the only assumption of bounded velocity (3.3). However, in this case we have Proposition 3.1 below that establishes a system which is not closed, since the pressure is not known at sufficiently high order of accuracy. Thus as in $[36,14]$, in parallel to $(3.3)$ we shall also consider the more restrictive case of slow velocities $U=O(\varepsilon)$, which leads to

$$
\begin{array}{rlrl}
U & =O(\varepsilon), & W=O\left(\varepsilon^{2}\right), \\
\partial_{X} U & =O(\varepsilon), & \partial_{X} W=O\left(\varepsilon^{2}\right), \\
\partial_{Z} U & =O(1), \quad \partial_{Z} W=O(\varepsilon) .
\end{array}
$$

In one or the other case, we have the following preliminary expansion.

Proposition 3.1 (Thin-layer asymptotics) Consider a solution to the viscoplastic model written in curvilinear coordinates (2.11)-(2.20), that includes a static/flowing transition (2.30). We make the thin-layer assumptions (3.1), (3.2), and we suppose that the deviatoric stress is only pure shear up to $\varepsilon^{2}$, which means that $\mathbf{\Sigma}+$ pId has $O\left(\varepsilon^{2}\right)$ diagonal terms. We assume either the scaling hypothesis (3.3) for bounded velocity, or (3.4) for slow velocity, and that $\partial_{Z} U$ is really of order $1 / \varepsilon$, respectively 1 (i.e. it is not smaller than this scale) in the flowing domain. We assume additionally that the viscosity is small enough,

$$
\nu=\left\{\begin{array}{l}
O\left(\varepsilon^{2}\right) \text { in case of the scaling (3.3), } \\
O(\varepsilon) \text { in case of the scaling (3.4). }
\end{array}\right.
$$


Then $U$ satisfies

$$
\partial_{t} U+U \partial_{X} U+W \partial_{Z} U+g \partial_{X}(B+h \cos \theta)-\partial_{Z}\left(\Sigma_{X Z}\right)=O\left(\varepsilon^{2}\right),
$$

where $\Sigma_{X Z}$ is given by

$$
\Sigma_{X Z}=\nu \partial_{Z} U+\kappa \operatorname{sgn}\left(\partial_{Z} U\right)+O\left(\varepsilon^{3}\right)
$$

in which $\operatorname{sgn}\left(\partial_{Z} U\right)$ has to be interpreted as multivalued, and

$$
\Sigma_{X Z}=O\left(\varepsilon^{3}\right) \quad \text { at } Z=h .
$$

The normal velocity $W$ is obtained by

$$
\partial_{X} U+\partial_{Z} W=O\left(\varepsilon^{2}\right), \quad W=0 \text { at } Z=0,
$$

and the width $h(t, X)$ of the layer obeys the mass conservation equation (2.14). The coefficient $\kappa$ is still given by (2.20).

Proof. Since $\partial_{Z} U$ is really of order $1 / \varepsilon$, respectively 1 , in the flowing domain, the expression (2.19) gives with the assumed orders of magnitude that

$$
\|D \mathbf{U}\|=\frac{1}{\sqrt{2}}\left|\partial_{Z} U\right|\left(1+O\left(\varepsilon^{2}\right)\right) \quad \text { for } Z>b(t, X) .
$$

Using the small viscosity assumption (3.5), the rheological relations (2.17) then give that $\Sigma_{X X}+p=O\left(\varepsilon^{2}\right), \Sigma_{Z Z}+p=O\left(\varepsilon^{2}\right)$ for $Z>b$. Our assumption of only pure shear up to $\varepsilon^{2}$ indeed means that these approximations are valid also in the static zone. Thus we have for $0 \leq Z \leq h$

$$
\Sigma_{X X}+p=O\left(\varepsilon^{2}\right), \quad \Sigma_{Z Z}+p=O\left(\varepsilon^{2}\right) .
$$

These properties imply that $\Sigma_{X X}-\Sigma_{Z Z}=O\left(\varepsilon^{2}\right)$, and with the scaling (3.2) we obtain from (2.15) the approximate boundary conditions at the free surface

$$
\Sigma_{X Z}=O\left(\varepsilon^{3}\right) \quad \text { at } Z=h, \quad \Sigma_{Z Z}=O\left(\varepsilon^{4}\right) \quad \text { at } Z=h .
$$

We then look for an approximation of (2.12) (momentum along the topography) up to $O\left(\varepsilon^{2}\right)$. Therefore we need an approximation of (2.13) (momentum normal to the topography) up to $O(\varepsilon)$. This latter equation is therefore expanded as

$$
\partial_{Z}\left(g Z \cos \theta-\Sigma_{Z Z}\right)=O(\varepsilon),
$$

from which we deduce with (3.12), (3.11)

$$
p=-\Sigma_{X X}+O\left(\varepsilon^{2}\right)=-\Sigma_{Z Z}+O\left(\varepsilon^{2}\right)=g \cos \theta(h-Z)+O\left(\varepsilon^{2}\right) .
$$

Reporting this in (2.12), we get (3.6). The normal velocity $W$ is deduced from the knowledge of $U$ by the incompressibility condition (2.11) and the bottom condition (2.16), that simplify to (3.9). We then write asymptotically the shear stress relation (2.18) by using (3.10), that gives (3.7) in the flowing part. Using the assumption of only pure shear up to $\varepsilon^{2}$ and the multivalued shear stress relation (2.18), we conclude that (3.7) is also valid in the static part, with $\operatorname{sgn}\left(\partial_{Z} U\right)$ being interpreted as multivalued. The boundary condition (3.8) comes from (3.12), and the mass conservation (2.14) and coefficient value (2.20) are kept unchanged.

The system (3.6)-(3.9), (2.14), (2.20) involves an unknown $U(t, X, Z)$ defined up to errors in $\varepsilon^{2}$. However, there are two main difficulties that arise. The first is that the value of $\kappa$ that appears in (3.7) needs to be known up to an error in $\varepsilon^{3}$. According to (2.20) this means to know the pressure $p$ up to an error in $\varepsilon^{3}$, what we do not have from the hydrostatic pressure approximation (3.14). Thus the system is not closed at the required order of approximation, and one needs to express non-hydrostatic corrections. The second difficulty is that it is not obvious how to describe the interface dynamics with (3.6), because of the inertial terms $U \partial_{X} U, W \partial_{Z} U$. Note however that the system is coherent with the scaling assumptions (3.2) and (3.3), because the formulas (3.14), (3.7) give $\boldsymbol{\Sigma}=O(\varepsilon)$, and all the terms in (3.6) are bounded. 


\section{Slow flow model}

In order to resolve the difficulties stated in the previous section on the thin-layer system (3.6)-(3.9), (2.14), (2.20), we complete now the analysis in the case of slow velocity, i.e. assumption (3.4). It is of course more restrictive than (3.3), but at the time we are not able to treat this latter case.

The first observation to make is that for this assumption (3.4) to remain valid for all time, we need that $\partial_{t} U=O(\varepsilon)$, since $U$ must be $O(\varepsilon)$. Looking thus at the velocity equation (3.6) and using that from (2.4) one has $d_{X} B=\sin \theta$, we get the compatibility condition

$$
g \sin \theta=\partial_{Z}\left(\Sigma_{X Z}\right)+O(\varepsilon) .
$$

In order to proceed, we make the small viscosity assumption

$$
\nu=O\left(\varepsilon^{2}\right),
$$

which is stronger than (3.5), but not too restrictive for applications (see Comment 3 after Theorem 4.1). Using the shear stress relation (3.7), the definition (2.20) of $\kappa$ in terms of the pressure, and the hydrostatic approximation (3.14), this leads to the relation in the flowing phase $g \sin \theta=-g \mu_{s} \cos \theta \operatorname{sgn}\left(\partial_{Z} U\right)+O(\varepsilon)$. If $\mu_{s}$ is not $O(\varepsilon)$, then also $\theta$ must not be small (not $O(\varepsilon)$ ), and we get the two conditions

$$
\mu_{s}=|\tan \theta|+O(\varepsilon)
$$

and

$$
\operatorname{sgn}\left(\partial_{Z} U\right)=-\operatorname{sgn}(\theta) \text { for } Z>b(t, X) .
$$

The condition (4.3) means that the effects of gravity and friction compensate, up to a fluctuation of order $\varepsilon$. Without this assumption, one of the two forces would dominate the other, with the effect of a strong acceleration/deceleration, violating our assumption $\partial_{t} U=O(\varepsilon)$.

With the previous assumptions we can close the system (3.6)-(3.9), (2.14), (2.20) and express a non-hydrostatic correction to the pressure.

Theorem 4.1 (Slow flow model) We make the assumptions of thin-layer (3.1), small viscosity (4.2), compensation at leading order of gravity and friction (4.3) (note that $\theta$ can nevertheless be $X$ dependent), and consider the regimes where

$$
\text { the pressure } p \text { is convex with respect to } Z \text { in }[0, h(t, X)] \text {. }
$$

Then the solutions $(U(t, X, Z), h(t, X), b(t, X))$ to the viscoplastic model written in curvilinear coordinates (2.11)-(2.20) that include a static/flowing transition (2.30) and that satisfy the scaling hypothesis (3.2), the deviatoric stress being only pure shear up to $\varepsilon^{2}$ (that is $\boldsymbol{\Sigma}+p \mathrm{I} d$ has $O\left(\varepsilon^{2}\right)$ diagonal terms), and (3.4) with $\partial_{Z} U$ really of order 1 (i.e. it is not smaller than this scale) in the flowing domain, are characterized by the mass conservation

$$
\partial_{t}\left(h-\frac{h^{2}}{2} d_{X} \theta\right)+\partial_{X}\left(\int_{0}^{h} U d Z\right)=0,
$$

the shear sign condition

$$
\operatorname{sgn}\left(\partial_{Z} U\right)=-\operatorname{sgn}(\theta) \quad \text { for } Z>b(t, X),
$$

the velocity equation

$\partial_{t} U+g\left(\sin \theta+\partial_{X}(h \cos \theta)\right)+\operatorname{sgn}(\theta) \partial_{Z}\left(\mu_{s} p\right)-\partial_{Z}\left(\nu \partial_{Z} U\right)=O\left(\varepsilon^{2}\right) \quad$ for $b(t, X)<Z<h(t, X)$, 
with non-hydrostatic pressure $p$ given by

$$
p=g\left(\cos \theta+\sin \theta \partial_{X} h-2|\sin \theta| \frac{\partial_{X} U}{\left|\partial_{Z} U\right|}\right) \times(h-Z)+O\left(\varepsilon^{3}\right), \quad \text { for } b(t, X)<Z<h(t, X),
$$

the boundary conditions

$$
\begin{gathered}
\nu \partial_{Z} U=O\left(\varepsilon^{3}\right) \text { at } Z=h(t, X), \\
U=0 \text { at } Z=b(t, X), \\
\nu \partial_{Z} U=0 \text { at } Z=b(t, X),
\end{gathered}
$$

and the static equilibrium condition

$$
g\left|\sin \theta+\partial_{X}(h \cos \theta)\right| \leq-\left(\partial_{Z}\left(\mu_{s} p\right)\right)_{b}+O\left(\varepsilon^{2}\right),
$$

where the index $b$ means that the quantity is evaluated at $Z=b(t, X)$.

Proof. Applying Proposition 3.1, we arrive at the system (3.6)-(3.9) with the mass conservation (2.14) (which is (4.6)), the definition (2.20) of $\kappa$, and as it has been proved above, (4.4) (i.e. (4.7)) must hold. According to the scaling (3.4), the inertial terms $U \partial_{X} U, W \partial_{Z} U$ in the velocity equation (3.6) are $O\left(\varepsilon^{2}\right)$, and can be neglected. We can then just skip the equation (3.9) that defines $W$.

We would like now to obtain an expansion of $p$ up to errors in $\varepsilon^{3}$, so that we could close the shear stress equation (3.7) with the definition (2.20) of $\kappa$ in terms of the pressure. Expanding the normal velocity equation (2.13) at higher order than previously in (3.13) yields

$$
\partial_{Z}\left(g Z \cos \theta-\Sigma_{Z Z}\right)=\partial_{X} \Sigma_{X Z}+O\left(\varepsilon^{2}\right) .
$$

But using (4.1) and the boundary condition (3.8) we get

$$
\Sigma_{X Z}=-g \sin \theta(h-Z)+O\left(\varepsilon^{2}\right),
$$

which gives in (4.14) by using that $d_{X} \theta=O(\varepsilon)$ from (3.1),

$$
\partial_{Z}\left(g Z \cos \theta-\Sigma_{Z Z}\right)=-g \sin \theta \partial_{X} h+O\left(\varepsilon^{2}\right) .
$$

With the boundary condition (3.12) we deduce

$$
\Sigma_{Z Z}=-g\left(\cos \theta+\sin \theta \partial_{X} h\right)(h-Z)+O\left(\varepsilon^{3}\right) .
$$

Independently, taking into account the diagonal rheological relations (2.17), the expansion of the shear rate (3.10), and the value of $\partial_{Z} W$ from (3.9), one has

$$
-\Sigma_{Z Z}-p=2 \kappa \frac{\partial_{X} U}{\left|\partial_{Z} U\right|}+O\left(\varepsilon^{3}\right), \quad \text { for } Z>b,
$$

thus with (4.17) we get

$$
p=g\left(\cos \theta+\sin \theta \partial_{X} h\right)(h-Z)-2 \kappa \frac{\partial_{X} U}{\left|\partial_{Z} U\right|}+O\left(\varepsilon^{3}\right), \quad \text { for } Z>b .
$$

To leading order, this quantity is nonnegative. Thus the value (2.20) of $\kappa$ becomes $\kappa=\mu_{s} p$, and since $\partial_{X} U /\left|\partial_{Z} U\right|=O(\varepsilon)$ according to the orders of magnitude (3.4), for $\kappa$ in (4.19) we only need to use the hydrostatic approximation $p=g \cos \theta(h-Z)+O\left(\varepsilon^{2}\right)$. We thus get

$$
p=g\left(\cos \theta+\sin \theta \partial_{X} h\right)(h-Z)-2 \mu_{s} g \cos \theta(h-Z) \frac{\partial_{X} U}{\left|\partial_{Z} U\right|}+O\left(\varepsilon^{3}\right), \quad \text { for } Z>b .
$$

With (4.3), we can replace then $\mu_{s}$ by $|\tan \theta|$, and we arrive at the stated pressure value (4.9). 
Then we write the velocity equation (3.6) by taking into account the shear stress value (3.7),

$$
\partial_{t} U+g\left(\sin \theta+\partial_{X}(h \cos \theta)\right)-\partial_{Z}\left(\nu \partial_{Z} U+\mu_{s} p \operatorname{sgn}\left(\partial_{Z} U\right)\right)=O\left(\varepsilon^{2}\right),
$$

and the boundary condition (3.8) that simplifies to $\nu \partial_{Z} U=O\left(\varepsilon^{3}\right)$ at $Z=h$, i.e. (4.10). We conclude that solutions to the two-dimensional viscoplastic model with static/flowing interface that have the orders of magnitude (3.2), (3.4) satisfy the system (4.6), (4.7), (4.21), (4.9), (4.10). We notice that the pressure in (4.9) is only defined in the flowing phase, because the ratio $\partial_{X} U / \partial_{Z} U$ is not defined in the static phase. Conversely, given a solution to the system (4.6), (4.7), (4.21), (4.9), (4.10) with intial data $U^{0}=O(\varepsilon)$, we see that we get a solution to the original viscoplastic model with the orders of magnitude (3.2), (3.4).

In order to go further, we need to take into account the assumption (4.5) of convexity of the pressure in the whole layer $[0, h]$. According to Lemma A.1 in Appendix, applied here with $\Phi=g\left(\sin \theta+\partial_{X}(h \cos \theta)\right)$, this assumption allows us to reformulate the problem (4.21) in the flowing phase only (thus avoiding the knowledge of the values of the pressure in the static phase), together with boundary conditions at the interface. This allows us to replace (4.21) by (4.8), (4.11)-(4.13), and concludes the proof of the Theorem.

The assumptions and statements in Theorem 4.1 deserve some comments.

1. The assumption (4.5) of convexity of the pressure seems to be rather valid in viscoplastic flows of interest as in the two-dimensional finite element simulations of [32], at least in its weaker form stated in (A.18), i.e. the function $\kappa(Z)$ is above its tangent at the interface $b$. As seen in the proof of Lemma A.1 in Appendix, this condition ensures the force balance in the static layer as soon as a condition holds at the interface. Without it the dynamics in the original viscoplastic system could show strong instabilities with creation of multiple flowing layers inside the static one. Thus (4.5) expresses a kind of condition of stability of the double layer configuration with flowing on top of static material.

2. In view of the pressure expansion (4.9) in the flowing layer, the convexity assumption (4.5) implies that $-\frac{\partial X U}{|\partial Z U|}(h-Z)$ is convex with respect to $Z \in(b, h)$, since this term is the only one that is nonlinear in $Z$. It is not easy to interpret this condition in terms of the velocity function $U(t, X, Z)$. This term and the linear one $g \sin \theta \partial_{X} h(h-Z)$ are the two non-hydrostatic corrections to the pressure.

3. The small viscosity assumption (4.2) induces a second-order term $-\partial_{Z}\left(\nu \partial_{Z} U\right)=O(\varepsilon)$ in the velocity equation (4.8), which is not negligible at the order of approximation of this equation. It is however smaller than the friction term $\partial_{Z}\left(\mu_{s} p\right)$, which is $O(1)$. This situation is characteristic of granular flows, where viscous effects are most of the time weaker than plastic effects. The orders of magnitude assumed in Theorem 4.1 and explained at the beginning of Section 3 are in particular satisfied in the experiments of [22], where the typical length is $L=1 \mathrm{~m}$, typical time $\tau=0.33$ s satisfying $L / \tau^{2}=g$, $h=0.02 \mathrm{~m}, \nu=5 \cdot 10^{-5} \mathrm{~m}^{2} \mathrm{~s}^{-1}$, leading to $\varepsilon \equiv h / L=0.02$, and the normalized viscosity $\nu \tau / L^{2} \simeq 10^{-5}$ is of the order of $\varepsilon^{2}$ or $\varepsilon^{3}$, thus eventually smaller than (4.2).

4. As in Proposition 2.2, the boundary conditions (4.11), (4.12) express the continuity of the velocity and shear stress across the interface. The reasons for these to hold are explained in Lemma A.1 in Appendix.

5. The static equilibrium condition (4.13) expresses that the shear stress $\Sigma_{X Z}$ in the static layer must be less than the yield stress $\kappa$ for this layer to remain static, as the proof of Lemma A.1 in Appendix shows. If this condition does not hold, the whole layer will flow down and we can solve the system by setting $b=0$, skipping the static equilibrium condition (4.13) and keeping only one of the two bottom conditions (4.11), (4.12). 
6. A particular solution to the slow flow model (4.6)-(4.13) is the simple shear flow of Subsection 2.3, for which there is no dependency in $X$. In the next two subsections we give some other particular solutions.

7. According to the interface condition (4.11) one has $U(t, X, b(t, X))=0$, thus differentiating with respect to $X$ we get

$$
\frac{\partial_{X} U}{\partial_{Z} U}(t, X, b(t, X))=-\partial_{X} b(t, X),
$$

where the ratio on the left-hand side has to be computed as the limit as $Z \rightarrow b(t, X)$ by above (since both the numerator and the denominator vanish in the case $\nu>0$ ). This gives the limit value at $Z=b$ of the pressure expansion (4.9).

\subsection{Rest equilibrium}

We provide here a particular solution to the slow model (4.6)-(4.13), in the fully static case. We thus look for a solution such that $U \equiv 0$. Then $b \equiv h,(4.9),(4.7),(4.8)$ say nothing, and (4.10)-(4.12) are satisfied. The mass equation (4.6) gives that $\partial_{t} h=0$. It remains to write the static equilibrium condition (4.13). Viewing the pressure value (4.9) as an expansion in $Z-b$ (recall that $b=h$ ) and using (4.22) yields the formal relation $\left(\partial_{Z} p\right)_{b}=-g\left(\cos \theta+\sin \theta \partial_{X} h-2 \sin \theta \partial_{X} b\right)+O\left(\varepsilon^{2}\right)$. Thus (4.13) reduces to

$$
\left|\sin \theta+\partial_{X}(h \cos \theta)\right| \leq \mu_{s}\left(\cos \theta+\sin \theta \partial_{X} h-2 \sin \theta \partial_{X} h\right)+O\left(\varepsilon^{2}\right),
$$

or equivalently

$$
\left|\frac{\sin \theta+\partial_{X}(h \cos \theta)}{\cos \theta-\partial_{X}(h \sin \theta)}\right| \leq \mu_{s}+O\left(\varepsilon^{2}\right) .
$$

The interpretation of this inequality is as follows. Consider the coordinates $(y(X), z(X))$ of a point at the free surface,

$$
y=x-h \sin \theta, \quad z=B+h \cos \theta,
$$

where $x$ is the horizontal coordinate, according to the geometrical relations (2.4), (2.5), see Figure 1. Then (4.24) says that $|d z / d y| \leq \mu_{s}+O\left(\varepsilon^{2}\right)$, i.e. the slope of the free surface is less than or equal to $\mu_{s}$. We conclude that this natural slope condition (4.24) is the one for the solution to the slow flow model to remain at rest. It is a first order correction taking into account the width $h$ and the dependency in $X$ to the simple shear condition (2.39). The rest equilibrium solution to the slow model is characterized by $U \equiv 0, b=h=h(X)$, satisfying the slope condition (4.24).

\subsection{Steady flows with static/flowing transition}

The fully static solution of the previous subsection does not include a static/flowing interface. We exhibit here some steady flows with static/flowing interface that are solutions of the slow flow model. A solution to (4.6)-(4.13) is built as follows, under the assumption that the viscosity is negligible,

$$
\nu=O\left(\varepsilon^{3}\right) .
$$

This assumption allows us to keep only the boundary condition $U(t, X, b(t, X))=0$ from (4.10)-(4.12). We assume that $\theta(X)$ has a constant sign, and we take the velocity of the form

$$
U(X, Z)=\widetilde{U}(Z-h(X))
$$

where $\widetilde{U}(Y)$ is an arbitrary continuous profile verifying

$$
\operatorname{sgn}(\theta) d \widetilde{U} / d Y \leq 0, \quad \widetilde{U}(Y)=0 \quad \text { for } Y \leq-y_{0},
$$


for some $y_{0} \geq 0$. We consider a spatial domain where $h(X) \geq y_{0}$. Then the mass equation (4.6) is verified since $\int_{0}^{h} U d Z=\int_{-\infty}^{0} \widetilde{U} d Y$ is independent of $X$, and the sign condition (4.7) holds according to (4.28). With the vanishing condition at the interface (4.11) we have $b(X)=h(X)+y_{1}$ with $y_{1}$ the largest value for which $\widetilde{U}\left(y_{1}\right)$ vanishes, thus $\partial_{X}(h-b)=0$, $\partial_{X} U / \partial_{Z} U=-\partial_{X} h$, and the presure value (4.9) becomes $p=g\left(\cos \theta-\sin \theta \partial_{X} h\right)(h-Z)+$ $O\left(\varepsilon^{3}\right)$. Thus the velocity equation (4.8) and the static equilibrium condition (4.13) give the single equation

$$
\sin \theta+\partial_{X}(h \cos \theta)-\mu_{s} \operatorname{sgn}(\theta)\left(\cos \theta-\partial_{X}(h \sin \theta)\right)=O\left(\varepsilon^{2}\right) .
$$

The boundary conditions (4.10), (4.12) are satisfied up to $O\left(\varepsilon^{3}\right)$. Thus there remains only the condition (4.29). With the change of variable (4.25), this means that $|d z / d y|=\mu_{s}+O\left(\varepsilon^{2}\right)$, i.e. the slope of the free surface is equal to $\mu_{s}$. The steady solution built in this way has free surface and static/flowing interface with slopes $\mu_{s}$, and it generalizes the steady simple shear solution given by $(2.45)$ with $|\tan \theta|=\mu_{s}$. The solution is illustrated on Figure 3 .

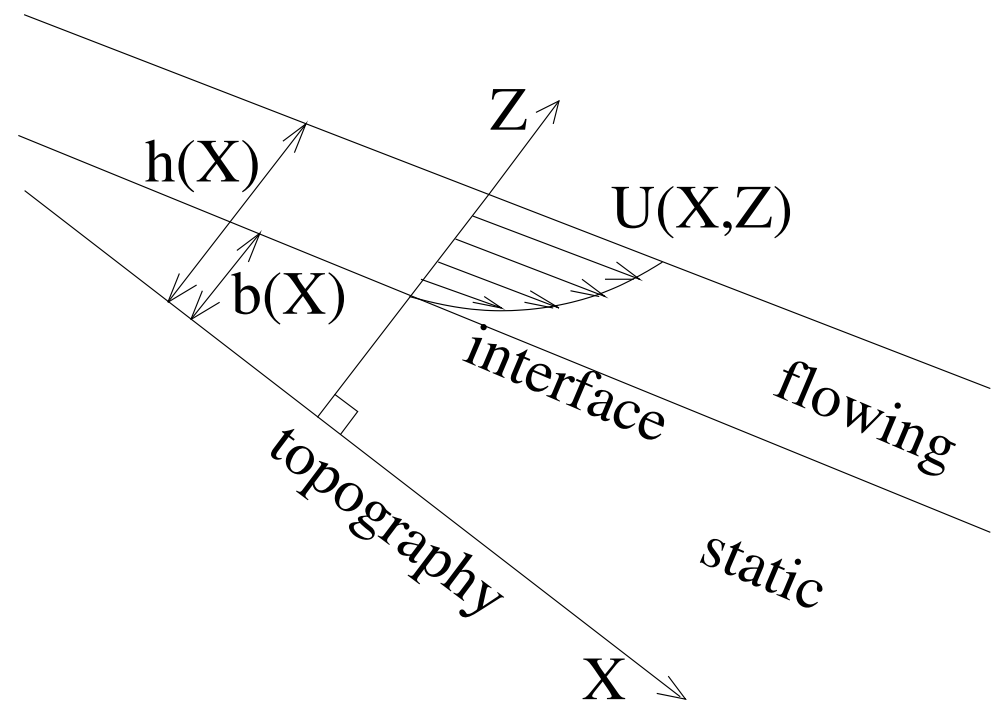

Figure 3: Steady flow with static/flowing transition, in the case when $\theta$ is constant. The topography has slope angle $\theta$. The free surface and the interface have slope $\mu_{s}$, so that gravity balances internal friction within the flowing layer. The velocity profile is continuous across the interface, but not necessarily differentiable since the viscosity vanishes. The shape of the profile is arbitrary, and can be exponential for example, as it is often observed in experiments.

Observing this picture, the reader may wonder if the situation represented, with $|\tan \theta|>$ $\mu_{s}$, is possible. The answer is yes. Indeed, what the static equilibrium condition (4.13) says here is only that the slope of the free surface is $\mu_{s}$, in contrast with the simple shear solution of Proposition 2.2, where the static equilibrium condition says (2.39), i.e. $|\tan \theta| \leq \mu_{s}$. Thus here there is no condition on $\theta$, and $|\tan \theta|$ can be less or greater than $\mu_{s}$. Note however that the asymptotic assumption (4.3) imposes that $|\tan \theta|$ is not far from $\mu_{s}$. The interpretation of the situation depicted here is that the flowing part of the material "does not feel the topography" through the static layer, similarly as in classical thin-layer (i.e. without friction) where the rest solution with horizontal free surface exists whatever the topography is. 


\subsection{Interface dynamics and discussion}

The slow flow problem (4.6)-(4.13) can be reformulated as follows. Defining $S(t, X, Z)$ by

$$
S=-g \operatorname{sgn}(\theta)\left(\sin \theta+\partial_{X}(h \cos \theta)\right)-\partial_{Z}\left(\mu_{s} p\right),
$$

the velocity equation (4.8) can be written (dropping the $\varepsilon$ ) as

$$
\partial_{t} U-\operatorname{sgn}(\theta) S-\partial_{Z}\left(\nu \partial_{Z} U\right)=0 \quad \text { for } Z>b(t, X),
$$

with the mass conservation (4.6), the sign condition (4.7), the pressure value (4.9), the boundary conditions (4.10)-(4.12), and the static condition (4.13), that becomes

$$
S(t, X, b(t, X)) \geq 0
$$

Note that in the simple shear case (no dependency in $X$ ), we recover the system obtained in Proposition 2.2. The pressure convexity assumption (4.5) gives that $\partial_{Z} S \leq 0$. One can prove by applying a formal maximum principle [41] that this ensures that the monotonicity condition (4.7) remains true if it is the case at initial time. Indeed, without (4.5), the solution $U$ to (4.31) would not remain monotone with respect to $Z$, violating the conditions under which it has been derived.

As in the simple shear situation, the three boundary conditions (4.10)-(4.12) (reducing to the single condition (4.11) in the inviscid case $\nu=0$ ) determine the dynamics of the static/flowing interface $b(t, X)$.

If $\nu=0$, differentiating the boundary condition $U(t, X, b(t, X))=0$ with respect to $t$ enables to get $\partial_{t} b(t, X) \partial_{Z} U(t, X, b(t, X))=-\partial_{t} U(t, X, b(t, X))$, and with (4.31) we obtain

$$
\partial_{t} b(t, X)=-\operatorname{sgn}(\theta) \frac{S(t, X, b(t, X))}{\partial_{Z} U(t, X, b(t, X))}, \quad \text { if } \partial_{Z} U(t, X, b(t, X)) \neq 0 .
$$

Using (4.32) and the sign condition (4.7) we obtain $\partial_{t} b \geq 0$ whenever $\partial_{Z} U(t, X, b(t, X)) \neq 0$. However, we can prove [41] that it is possible to have $\partial_{t} U<0$ together with $\partial_{Z} U(t, X, b(t, X))=$ 0 and $S(t, X, b(t, X))=0$. Hence, the formula (4.33) represents only partly the dynamics (because of the restrictions $\partial_{Z} U(t, X, b(t, X)) \neq 0$ and $\partial_{t} b \geq 0$ ), and it is not possible to formulate the evolution of the interface by specifying a general simple formula for $\partial_{t} b$. Instead we have to use the whole formulation with the source definition (4.30), the velocity equation (4.31), the mass conservation (4.6), the sign condition (4.7), the pressure value (4.9), the boundary conditions (4.10)-(4.12), and the static condition (4.32). Specific numerical methods have then to be used, see [40]. Noticing the hydrostatic approximation $p=g \cos \theta(h-Z)+O\left(\varepsilon^{2}\right)$ from (3.14), and the approximation $S=-g|\sin \theta|-\mu_{s} \partial_{Z} p+O(\varepsilon)$ from (4.30), we get $S=g \cos \theta\left(\mu_{s}-|\tan \theta|\right)+O(\varepsilon)$. With the assumption (4.3) of compensation of gravity and friction, this is $O(\varepsilon)$, but anyway the formula (4.33) with this approximation of $S$ identifies with the $\partial_{t} b$ equation of the models discussed in [14], namely the model equation (4.22) in that reference, the BCRE model ((4.16) in [14]) from [10], and Khakhar's model ((4.14) in [14]) from [36]. In order to improve the expansion we need here to write down in (4.30) the next terms from the pressure expansion (4.9). The term in $\partial_{X} h$ corresponds in particular to the ones in (4.5) or (4.18) in [14]. However, the term in $\partial_{X} U / \partial_{Z} U$ in (4.9) is specific to our approach, it includes a non-hydrostatic coupling that cannot be expressed by averages in $Z$. A description of physically relevant static/flowing interface evolution equations is provided in [34], in the context of thin-layer models. We remark that in this description, no term in $\partial_{X} h$ is present, and no interface evolution is possible if the density is constant, contrarily to our model. Moreover, the velocity is discontinuous across the interface, contrarily to our case where it is continuous, with first derivative continuous in the viscous case.

We conclude that on one hand our approach here is quantitatively close to the BCRE and Khakhar models, and on the other hand its formulation (4.6)-(4.13) is better mathematically, it is in closed form and holds without restrictions like $\partial_{Z} U \neq 0$ in (4.33). In comparison to the 
BCRE and Khakhar models it includes additional terms proportional to $\partial_{X} h$ in (4.8), (4.9) and a higher-order nonlinear coupling term in the pressure. Our model has some remarkable particular steady solutions with free surface slope equal to or less than the friction coefficient $\mu_{s}$. It can include the case of a single flowing layer without static phase, and the dynamic transition from the two-layer situation to the single-layer one, according to Comment 5 after Theorem 4.1. Some clear numerical schemes can be derived [40] (at least in the case of uncoupled source $S$, i.e. not depending on $U$ or its derivatives), including the case of non-zero viscosity. A comparison of the simple shear approximation of Subsection 2.3 with experimental data is performed in [40], showing the relevance of our formulation, including the effect of viscosity.

The effect of the $Z$ dependency of $S$ is described in the inviscid case in [41], showing that the eventual zero $b^{*}(t, X)$ of $S$ in the variable $Z$ (i.e. satisfying $S\left(t, X, b^{*}(t, X)\right)=0$ ) merely drives the evolution of $b(t, X)$. This property enables to put into motion the static phase (i.e. the decrease of $b$ ) even without viscosity, which is not possible without dependency in $Z$, as seen in the explicit solution (2.45), (2.46). Therefore, in order to describe the fact that the static phase can be put into motion, for example in the case of horizontal space inhomogeneities and initially vanishing velocity, we have to take into account the $Z$ dependency of the source $S$, which means to include the coupling with the pressure expansion (4.9) with the term in $\partial_{X} U / \partial_{Z} U$, that produces the $Z$ dependency of $S$. The effect of this term is illustrated by the configurations of Subsections 4.1 and 4.2 , where $\partial_{X} U / \partial_{Z} U=-\partial_{X} h$ is non-zero.

When considering the full coupling with the source (4.30) and the pressure formula (4.9), we have first to remark that the model is only valid as long as the pressure remains convex with respect to $Z$, a condition that could eventually break up in finite time. The coupled problem (4.31), (4.30) with $p$ given by (4.9) looks difficult to solve. It is nonlinear in the two space derivatives of $U$, because of the ratio $\partial_{X} U / \partial_{Z} U$ in (4.9). It is possible to remove the infinite values of this ratio, by replacing $\partial_{X} U /\left|\partial_{Z} U\right|$ in (4.9) by $\partial_{X} U /\left(\left(\partial_{Z} U\right)^{2}+4\left(\partial_{X} U\right)^{2}\right)^{1 / 2}$, without affecting the accuracy of the approximation. Indeed the denominator $\left|\partial_{Z} U\right|$ has been obtained as an expansion of $\|D \mathbf{U}\|$ in (2.19). However by doing this we loose the nice relation (4.22). The equation anyway contains second-order terms linear in $\partial_{X Z}^{2} U\left(\right.$ and $\partial_{Z Z}^{2} U$ ), that make it ill-posed (it is like the backward heat equation) unless adding sufficient viscosity in $X$ (and eventually in $Z$ ). This ill-posedness is probably related to the ill-posedness established in [8] for the initial viscoplastic model in the regime close to stopping. The formulation and simulation of a well-posed suitably modified system with $X$ dependency is an important issue that will be explored in future works.

\section{Conclusion}

The description of avalanche flows of granular materials involves the key feature of the dynamics of the static/flowing interface. In contrast with the usual approach which is to formulate a phenomenological differential equation on the interface, we have been able to derive analytically a set of equations for the velocity, the width of the domain, and the interface, from a thin-layer asymptotics starting from an incompressible viscoplastic model with DruckerPrager yield stress. In contrast to the so-called depth-averaged models, our model keeps the normal to the flow variable, and is set in the flowing phase as a parabolic-like model with formally overdetermined boundary conditions that express the continuity of velocity and shear stress across the interface. The extra condition determines the dynamics of the interface, but cannot be replaced by a simple ordinary differential equation on the position of the interface. The model is quantitatively close to the BCRE and Khakhar models, but at the same time its formulation is more coherent mathematically since it is a closed system with two space variables without restriction on the velocity slope or on the shape of the velocity. Moreover it can take into account small, but non-zero, viscosity. A specific property is that it involves a non-hydrostatic nonlinear coupling term in the pressure, that could have the effect of displacing the static/flowing interface according to the space inhomogeneities. 
The model is described in Theorem 4.1 by the system (4.6)-(4.13). A synthetic equivalent formulation is to use the source definition (4.30), then the velocity equation (4.8) becomes (4.31), and the static equilibrium condition (4.13) becomes (4.32). The model is established under the conditions that the curvature of the topography is small (3.1) the internal friction angle is close to the slope angle (4.3), the velocity is small (3.4), the viscosity is small (4.2), and the pressure is convex (4.5).

The evaluation of the model in its full generality needs to be explored.

\section{Appendix: Formulation in the flowing phase with extra boundary condition}

In this appendix we would like to justify conditions under which we have the mathematical equivalence between multivalued equations set in the whole material and equations set in the flowing phase only, with extra boundary condition coming from the continuity of the velocity and shear stress across the interface.

We consider an unknown $U(t, X, Z)$ defined for $0<Z<h(t, X)$, where $h(t, X)>0$ is assumed to be known. Given a source $\Phi(t, X)$ independent of $Z$, the problem is set as

$$
\partial_{t} U+\Phi-\partial_{Z}\left(\nu \partial_{Z} U+\kappa \operatorname{sgn}\left(\partial_{Z} U\right)\right)=0, \quad \text { for } 0<Z<h(t, X),
$$

where the sign is understood as multivalued (i.e. it can take any value in $[-1,1]$ at all locations $(t, X, Z)$ where $\left.\partial_{Z} U=0\right), \nu \geq 0$ and $\kappa(t, X, Z)$ are given. We complete the problem with the boundary condition

$$
\nu \partial_{Z} U+\kappa \operatorname{sgn}\left(\partial_{Z} U\right)=0 \quad \text { at } Z=h(t, X),
$$

and the no slip condition at the bottom $U=0$ at $Z=0$. This condition is however unnecessary since we are looking for solutions to (A.1) that have a static/flowing interface $0<b(t, X)<h(t, X)$ such that

$$
\begin{gathered}
U(t, X, Z)=0 \text { for } 0<Z<b(t, X) \text { (static layer), } \\
\partial_{Z} U(t, X, Z) \neq 0 \quad \text { for } b(t, X)<Z<h(t, X) \text { (flowing layer). }
\end{gathered}
$$

Lemma A.1 (Formulation with extra boundary condition) Assume that $\kappa(t, X, Z)$ is continuous with respect to $Z \in[0, h]$ and satisfies

$$
\begin{gathered}
\kappa(t, X, h(t, X))=0, \quad \kappa(t, X, Z)>0 \text { for } 0<Z<h, \\
\kappa \text { is convex with respect to } Z \text { in }[0, h] .
\end{gathered}
$$

Assume given the sign of the shear $s_{b}= \pm 1$, satisfying

$$
s_{b} \Phi \leq 0
$$

Then the problem of finding a solution $U(t, X, Z)$ defined for $0<Z<h(t, X)$ to the multivalued problem (A.1)-(A.3) with static/flowing interface $b(t, X) \in(0, h(t, X))$ is equivalent to finding a solution $U(t, X, Z)$ defined in the flowing layer $b(t, X)<Z<h(t, X)$ only, to

$$
\begin{gathered}
s_{b} \partial_{Z} U>0 \text { for } Z>b, \\
\partial_{t} U+\Phi-s_{b} \partial_{Z} \kappa-\partial_{Z}\left(\nu \partial_{Z} U\right)=0 \text { for } Z>b,
\end{gathered}
$$

with the boundary conditions (the "+" meaning that the limit is taken by above)

$$
\nu \partial_{Z} U=0 \text { at } Z=h,
$$




$$
\begin{gathered}
U \rightarrow 0, \text { as } Z \rightarrow b+, \\
\nu \partial_{Z} U \rightarrow 0 \text { as } Z \rightarrow b+,
\end{gathered}
$$

and the static equilibrium condition

$$
-\left(\partial_{Z} \kappa\right)_{b} \geq|\Phi|,
$$

where the index $b$ means that the quantity is evaluated at $Z=b(t, X)$.

Proof. In this problem, the variable $X$ is only a parameter, and the dynamics is in the variables $t, Z$. We shall therefore omit the variable $X$. We use the notation

$$
\Sigma_{X Z}=\nu \partial_{Z} U+\kappa \operatorname{sgn}\left(\partial_{Z} U\right)
$$

A solution to (A.1)-(A.3) for some $b \in(0, h)$ can be characterized by three types of equations: equations in the static phase $Z<b$, equations in the flowing phase $Z>b$, and relations through the interface $Z=b$. These can be written as follows.

1. Interface jump relations. Assuming that $U$ does not jump through the interface (this is mandatory when viscosity is present), i.e. (A.10) holds, the equation (A.1) yields the property that the shear stress $\Sigma_{X Z}$ defined by (A.13) does not jump through the interface $Z=b(t)$. We shall denote its value by $\left(\Sigma_{X Z}\right)_{b(t)}$, or simply $\left(\Sigma_{X Z}\right)_{b}$. Then the relation (A.13) implies that $\kappa \operatorname{sgn}\left(\partial_{Z} U\right)$ does not jump through the interface (if $\nu=0$ it is obvious, and in the case $\nu>0$, use that the relation (A.13) with (A.4) gives a Lipschitz continuous dependency of $\partial_{Z} U$ in terms of $\Sigma_{X Z}$ ). We shall call the value of $\kappa \operatorname{sgn}\left(\partial_{Z} U\right)$ at the interface by $\kappa_{b} s_{b}$. Taking the limit from the flowing zone yields indeed that $s_{b}= \pm 1$ is the sign of the shear $\partial_{Z} U$ in the flowing zone, i.e. (A.7). From (A.13) again, we get by difference that $\nu \partial_{Z} U$ is also continuous through the interface. We conclude that (A.11) holds. Moreover, we have the relation $\left(\Sigma_{X Z}\right)_{b}=\kappa_{b} s_{b}$.

2. Equation in the static phase. There, call $s(t, Z)=\operatorname{sgn}\left(\partial_{Z} U\right)$. Then (A.13) gives that

$$
\Sigma_{X Z}=\kappa s \text { for } 0<Z<b .
$$

But (A.1) with the value of $\Sigma_{X Z}$ at $b$ given by $\left(\Sigma_{X Z}\right)_{b}=\kappa_{b} s_{b}$ yields

$$
\Sigma_{X Z}=\kappa_{b} s_{b}-(b-Z) \Phi \text { for } 0<Z<b .
$$

It remains then to state with (A.14), (A.15) that $|s| \leq 1$. With (A.14) it is equivalent to write $\kappa-s_{b} \Sigma_{X Z} \geq 0$ and $\kappa+s_{b} \Sigma_{X Z} \geq 0$. Using the expression (A.15) of $\Sigma_{X Z}$, we are led to

$$
\begin{aligned}
& \kappa(t, Z)-s_{b}\left(\kappa_{b} s_{b}-(b-Z) \Phi\right) \geq 0 \text { for } 0<Z<b, \\
& \kappa(t, Z)+s_{b}\left(\kappa_{b} s_{b}-(b-Z) \Phi\right) \geq 0 \text { for } 0<Z<b .
\end{aligned}
$$

Since $\kappa \geq 0$, the second inequality holds because of assumption (A.6). With (A.15) it implies that $s_{b} \Sigma_{X Z}>0$ for $Z<b$, and with (A.14) that $s_{b} s>0$ for $Z<b$. According to the assumption (A.5), the left-hand side of the first line of (A.16) is convex with respect to $Z$. Since it vanishes at $Z=b$, we have to write that the derivative with respect to $Z$ at b is nonpositive, which leads to

$$
\left(\partial_{Z} \kappa\right)_{b}-s_{b} \Phi \leq 0 .
$$

With (A.6), this is equivalent to (A.12). Note in passing that the convexity assumption (A.5) could be replaced here by the weaker one

$$
\text { the function } \kappa(Z) \text { is above its tangent at } b .
$$

3. Equation in the flowing phase. Knowing that there the sign condition (A.7) holds, the relation (A.13) in the flowing zone $Z>b$ gives $\Sigma_{X Z}=\nu \partial_{Z} U+\kappa s_{b}$. Plugging this in (A.1) yields (A.8), while with (A.4) the boundary condition (A.2) becomes (A.9). 
The previous developments prove that if we have a solution to (A.1)-(A.3), then it solves (A.7)-(A.12). Conversely, the same arguments enable to prove that (A.7)-(A.12) implies (A.1)-(A.3), the details are omitted.

We can remark that the static equilibrium condition (A.12) does not involve the unknown velocity $U$, but only the interface position $b$ (which is also an unknown of the problem). As is proved above, if (A.12) is violated, one cannot find a shear stress $\Sigma_{X Z}$ satisfying the equations in the static zone. The interpretation is that in this situation, the solution to (A.1)-(A.2), if it exists, has no static phase.

The interesting property in the final formulation in the flowing phase is that we have three boundary conditions (A.9), (A.10), (A.11) (respectively one condition if $\nu=0$ ) for a parabolic (respectively hyperbolic) problem, thus one extra condition than expected in problems with fixed boundary. This extra condition determines implicitly the dynamics of the static/flowing interface $b(t, X)$.

\section{Acknowledgments}

This work has been partially funded by the ANR contract ANR-06-BLAN-0414 PLANETEROS, the ANR contract ANR-11-BS01-0016 LANDQUAKES, and the ERC contract ERCCG-2013-PE10-617472 SLIDEQUAKES.

\section{References}

[1] C. Acary-Robert, E.D. Fernández-Nieto, G. Narbona-Reina, P. Vigneaux, A wellbalanced finite volume-augmented Lagrangian method for an integrated Herschel-Bulkley model, J. Scientific Comput. 53 (2012), 608-641.

[2] C. Ancey, Plasticity and geophysical flows: A review, J. Non-Newtonian Fluid Mech. 142 (2007), 4-35, In Viscoplastic fluids: From theory to application.

[3] A. Aradian, E. Raphael, P.-G. De Gennes, Surface flow of granular materials: a short introduction to some recent models, C. R. Phys. 3 (2002), 187-196.

[4] I.S. Aranson, L.S. Tsimring, Continuum theory of partially fluidized granular flows, Phys. Rev. E 65 (2002), 061303.

[5] I.S. Aranson, L.S. Tsimring, F. Malloggi, E. Clement, Nonlocal rheological properties of granular flows near a jamming limit, Phys. Rev. E 78 (2008), 031303.

[6] N.J. Balmforth, R.V. Craster, A consistent thin-layer theory for Bingham plastics, J. Non-Newtonian Fluid Mech. 84 (1999), 65-81.

[7] N.J. Balmforth, R.V. Craster, R. Sassi, Shallow viscoplastic flow on an inclined plane, J. Fluid Mech. 470 (2002), 1-29.

[8] T. Barker, D.G. Schaeffer, P. Bohorquez, J.M.N.T. Gray, Well-posed and ill-posed behaviour of the $\mu(I)$-rheology for granular flow, J. Fluid Mech. 779 (2015), 794-818.

[9] G. Bayada, L. Chupin, S. Martin, Viscoelastic fluids in a thin domain, Quarterly of Applied Mathematics 65 (2007), 625-652.

[10] J.-P. Bouchaud, M.E. Cates, J.R. Prakash, S.F. Edwards, A model for the dynamics of sandpile surface, J. Phys. Paris I 4 (1994), 1383-1410.

[11] F. Bouchut, S. Boyaval, A new model for shallow viscoelastic fluids, Math. Models and Meth. in Appl. Sci. 23 (2013), 1479-1526. 
[12] F. Bouchut, S. Boyaval, Unified derivation of thin-layer reduced models for shallow freesurface gravity flows of viscous fluids, European J. Mech. - B/Fluids 55 (2016), 116-131.

[13] F. Bouchut, R. Eymard, A. Prignet, Convergence of conforming approximations for inviscid incompressible Bingham fluid flows and related problems, J. Evolution Eq. 14 (2014), 635-669.

[14] F. Bouchut, E.D. Fernández-Nieto, A. Mangeney, P.-Y. Lagrée, On new erosion models of Savage-Hutter type for avalanches, Acta Mech. 199 (2008), 181-208.

[15] F. Bouchut, A. Mangeney-Castelnau, B. Perthame, J.-P. Vilotte, A new model of Saint Venant and Savage-Hutter type for gravity driven shallow water flows, C.R. Acad. Sci. Paris, série I 336 (2003), 531-536.

[16] T. Boutreux, E. Raphael, P.-G. DeGennes, Surface flows of granular materials: a modified picture for thick avalanches, Phys. Rev. E 58 (1998), 4692-4700.

[17] D. Bresch, E.D. Fernández-Nieto, I.R. Ionescu, P. Vigneaux, Augmented Lagrangian Method and Compressible Visco-Plastic Flows : Applications to Shallow Dense Avalanches, Advances in Mathematical Fluid Mechanics, New Directions in Mathematical Fluid Mechanics, Springer, (2010), 57-89.

[18] J. Chauchat, M. Médale, A three-dimensional numerical model for dense granular flows based on the $\mu(I)$ rheology, J. Comput. Phys. 256 (2014), 696-712.

[19] G.B. Crosta, S. Imposimato, D. Roddeman, Numerical modeling of 2-D granular step collapse on erodible and nonerodible surface, J. Geophys. Res. Earth Surface 114 (2009), F03020.

[20] D.C. Drucker, W. Prager, Soil mechanics and plastic analysis or limit design, Quarterly of Applied Mathematics 10 (1952), 157-165.

[21] G. Duvaut, J.-L. Lions, Les inéquations en mécanique et en physique, Dunod, 1972.

[22] M. Farin, A. Mangeney, O. Roche, Fundamental changes of granular flow dynamics, deposition, and erosion processes at high slope angles: insights from laboratory experiments, J. Geophys. Res. Earth Surface 119 (2014), 504-532.

[23] E. D. Fernández-Nieto, J.M. Gallardo, P. Vigneaux, Efficient numerical schemes for viscoplastic avalanches. Part 1: the 1D case, J. Comput. Phys. 264 (2014), 55-90.

[24] E. D. Fernández-Nieto, P. Noble, J.-P. Vila, Shallow water equations for non newtonian fluids, J. Non-Newtonian Fluid Mech. 165 (2010), 712-732.

[25] Y. Forterre, O. Pouliquen, Flows of dense granular media, Ann. Rev. Fluid Mech. 40 (2008), 1-24.

[26] J.M.N.T. Gray, Granular flow in partially filled slowly rotating drums, J. Fluid Mech. 441 (2001), 1-29.

[27] J.M.N.T. Gray, A.N. Edwards, A depth-averaged $\mu(I)$-rheology for shallow granular freesurface flows, J. Fluid Mech. 755 (2014), 503-534.

[28] A.J. Holyoake, J.N. McElwaine, High-speed granular chute flows, J. Fluid Mech. 710 (2012), 35-71.

[29] O. Hungr, S.G. Evans, M.J. Bovis, J.N. Hutchinson, Review of the classification of landslides of the flow type, Environ. Eng. Geosci. 7 (2001), 221-238.

[30] I.R. Ionescu, Onset and dynamic shallow flow of a viscoplastic fluid on a plane slope, J. Non-Newtonian Fluid Mech. 165 (2010), 1328-1341. 
[31] I.R. Ionescu, Viscoplastic shallow flow equations with topography, J. Non-Newtonian Fluid Mech. 193 (2013), 116-128.

[32] I.R. Ionescu, A. Mangeney, F. Bouchut, O. Roche, Viscoplastic modeling of granular column collapse with pressure-dependent rheology, J. Non-Newtonian Fluid Mech. 219 (2015), 1-18.

[33] R.M. Iverson, M. Reid, M. Logan, R. LaHusen, J.W. Godt, J. Griswold, Positive feedback and momentum growth during debris-flow entrainment of wet bed sediment, Nat. Geosci. 4 (2011), 116-121.

[34] R.M. Iverson, C. Ouyang, Entrainment of bed material by Earth-surface mass flows: review and reformulation of depth-integrated theory, Rev. Geophys. 53 (2015), 27-58.

[35] P. Jop, Y. Forterre, O. Pouliquen, A constitutive law for dense granular flows, Nature 441 (2006), 727-730.

[36] D.V. Khakhar, A.V. Orpe, P. Andresen, J.M. Ottino, Surface flow of granular materials: model and experiments in heap formation, J. Fluid Mech. 441 (2001), 225-264.

[37] P.-Y. Lagrée, L. Staron, S. Popinet, The granular column collapse as a continuum: validity of a two-dimensional navier-stokes model with a $\mu(I)$-rheology, J. Fluid Mech. 686 (2011), 378-408.

[38] C.H. Lee, Z.H. Huang, Y.M. Chiew, A three-dimensional continuum model incorporating static and kinetic effects for granular flows with applications to collapse of a two-dimensional granular column, Phys. Fluids 27 (2015), 113303.

[39] J.-L. Lions, Remarks on some nonlinear evolution problems arising in Bingham flows, Proceedings of the International Symposium on Partial Differential Equations and the Geometry of Normed Linear Spaces (Jerusalem, 1972), vol. 13, 155-172 (1973).

[40] C. Lusso, F. Bouchut, A. Ern, A. Mangeney, A simplified model for static/flowing dynamics in thin-layer flows of granular materials with yield, hal-00992309, 2015.

[41] C. Lusso, F. Bouchut, A. Ern, A. Mangeney, Explicit solutions to a free boundary model for the static/flowing transition in granular flows, hal-01180686, 2015.

[42] A. Mangeney, L.S. Tsimring, D. Volfson, I.S. Aranson, F. Bouchut, Avalanche mobility induced by the presence of an erodible bed and associated entrainment, Geophys. Res. Lett. 34 (2007), L22401.

[43] A. Mangeney, O. Roche, O. Hungr, N. Mangold, G. Faccanoni, A. Lucas, Erosion and mobility in granular collapse over sloping beds, J. Geophys. Res. Earth Surface115 (2010), F03040.

[44] GDR-MiDi, On dense granular flows, Eur. Phys. J. E Soft Matter 14 (2004), 341-365.

[45] G. Narbona-Reina, D. Bresch, On a shallow water model for non-newtonian fluids, Numerical Mathematics and Advanced Applications 2009 (Gunilla Kreiss, Per Lötstedt, Axel Moalqvist, and Maya Neytcheva, eds.), Springer Berlin Heidelberg, 2010, 693-701.

[46] P. Richard, A. Valance, J.-F. Métayer, P. Sanchez, J. Crassous, M. Louge, R. Delannay, Rheology of confined granular flows: scale invariance, glass transition, and friction weakening, Physical Review Letters 101 (2008), 248002.

[47] S.B. Savage, K. Hutter, The motion of a finite mass of granular material down a rough incline, J. Fluid Mech. 199 (1989), 177-215.

[48] D.G. Schaeffer, Instability in the evolution equations describing incompressible granular flow, J. Differential Equations 66 (1987), 19-50. 
[49] B. Sovilla, P. Burlando, P. Bartelt, Field experiments and numerical modeling of mass entrainment in snow avalanches, J. Geophys. Res. Earth Surface 111 (2006), F03007.

[50] Y.C. Tai, C.Y. Kuo, A new model of granular flows over general topography with erosion and deposition, Acta Mech. 199 (2008), 71-96. 